\title{
Brain Derived Neurotrophic Factor Modification of Epilep- tiform Burst Discharges in a Temporal Lobe Epilepsy Model
}

Sanaz Eftekhari ${ }^{1,2}$, Soraya Mehrabi ${ }^{3}$, Fariba Karimzadeh ${ }^{1}$, Mohammad-Taghi Joghataei ${ }^{1,2,4}$, Mojtaba Khaksarian ${ }^{5}$, Mahmoud Reza Hadjighassem $^{6,7}$, Majid Katebi ${ }^{8}$, Mansooreh Soleimani ${ }^{1,4}$

1. Cellular and Molecular Research Center, Iran University of Medical Sciences, Tehran, Iran.

2. Department of Neuroscience, School of Advanced Technologies in Medicine, Iran University of Medical Sciences, Tehran, Iran.

3. Shefa Neuroscience Center, Khatam-Alanbia Hospital, Tehran, Iran.

4. Department of Anatomy, School of Medicine, Iran University of Medical Sciences, Tehran, Iran.

5. Department of Physiology, School of Medicine, Lorestan University of Medical Sciences, Khorramabad, Iran

6. Department of Neuroscience, School of Advanced Technologies in Medicine, Tehran University of Medical Sciences, Tehran, Iran.

7. Brain and Spinal Cord Injury Research Centre, Imam Khomeini Hospital, Tehran University of Medical Sciences, Tehran, Iran.

8. Department of Anatomy, School of Medicine, Hormozgan University of Medical Sciences, Bandar Abbas, Iran

Citation: Eftekhari, S., Mehrabi, S., Karimzadeh, F., Joghataei, M. T., Khaksarian, M., Hadjighassem, M. R., et al. (2016). Brain derived neurotrophic factor Modification of epileptiform burst discharges in a temporal lobe epilepsy model. Basic and Clinical Neuroscience, 7(2), 115-120. http://dx.doi.org/10.15412/J.BCN.03070205

do: $h$ http://dx.doi.org/10.15412/J.BCN.03070205

Article info:

Received: 28 March 2015

First Revision: 19 April 2015

Accepted: 18 July 2015

Key Words:

Temporal lobe epilepsy, BDNF, EEG, Brain, Rat

\begin{abstract}
A B S T RA C T
Introduction: Transforming Growth Factor-Beta 1 (TGF- $\beta 1)$ is a pleiotropic cytokine with potent anti-inflammatory property, which has been considered as an essential risk factor in the inflammatory process of Ischemic Stroke (IS), by involving in the pathophysiological progression of hypertension, atherosclerosis, and lipid metabolisms. -509C/T TGF- $\beta 1$ gene polymorphism has been found to be associated with the risk of IS. The aim of this meta-analysis was to provide a relatively comprehensive account of the relation between $-509 \mathrm{C} / \mathrm{T}$ gene polymorphisms of TGF- $\beta 1$ and susceptibility to IS

Methods: Male Wistar rats were divided into sham (receiving phosphate buffered saline within dorsal hippocampus), pilocarpine (epileptic model of TLE), single injection BDNF (epileptic rats which received single high dose of BDBF within dorsal hippocampus), and multiple injections BDNF (epileptic rats which received BDNF in days 10,11, 12, and 13 after induction of TLE) groups. Their electrocorticogram was recorded and amplitude, frequency, and duration of spikes were evaluated.

Results: Amplitude and frequency of epileptiform burst discharges were significantly decreased in animals treated with BDNF compared to pilocarpine group.

Conclusion: Our findings suggested that BDNF may modulate the epileptic activity in the animal model of TLE. In addition, it may have therapeutic effect for epilepsy. More studies are necessary to clarify the exact mechanisms of BDNF effects.
\end{abstract}

\footnotetext{
* Corresponding Author:

Mansooreh Soleimani, $P h D$

Address: Cellular and Molecular Research Center, Iran University of Medical Sciences, Tehran, Iran

Tel: +98 (21) 88622578
}

E-mail: mansourehsoleimani@gmail.com 


\section{Introduction}

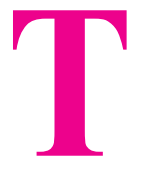

emporal Lobe Epilepsy (TLE), the most common form of partial epilepsy (Engel, 2001), is poorly controlled by current antiepileptic drugs (Cohen, Navarro, Duigou, \& Miles, 2003). The pathogenesis of TLE is still unclear. However, it has been shown that changes in the function of GABAergic signaling play an important role in the pathogenesis of TLE seizures (Cohen et al., 2003). $\gamma$-Aminobutyric acid (GABA), the main inhibitory neurotransmitter in the adult central nervous system (CNS), at the early stages of brain development can depolarize target cells by an outflow of chloride ion, $\mathrm{Cl}^{-}$. Immature neurons with a large amount of intracellular $\mathrm{Cl}^{-}$could be depolarized by releasing GABA because of chloride efflux (BenAri et al., 2007). Two cation-chloride cotransporters regulate flow of neuronal $\mathrm{Cl}^{-}: 1$ ) The $\mathrm{Na}-\mathrm{K}-2 \mathrm{Cl}$ cotransporters (NKCC1) loads neurons with $\mathrm{Cl}^{-}$and depolarize them followed by the release of GABA; 2) The $\mathrm{K}-\mathrm{Cl}$ cotransporter (KCC2) normally extrudes $\mathrm{Cl}^{-}$and hyperpolarize neurons followed by the release of GABA (Ben-Ari, Gaiarsa, Tyzio, $\&$ Khazipov, 2007). In the adult brain, under pathophysiological conditions such as epilepsy and trauma, alterations in the balance of NKCC1 and KCC2 activity may determine the hyperpolarizing, or depolarizing effects of GABA (Payne et al., 2003).

Various lines of evidence correlate epileptogenesis with altered functional expression of $\mathrm{NKCC} 1$ and $\mathrm{KCC} 2$ transporters. It has been demonstrated that animals deficient in $\mathrm{KCC} 2$ exhibit frequent generalized seizures and die shortly after birth (Woo et al., 2002). Increase of NKCC1 mRNA level in the piriform cortex of the rat amygdalakindling model of epilepsy has also been showed (Okabe et al., 2002). In addition, quantitative RT-PCR analyses of specimens taken from the subiculum of patients with drugresistant temporal lobe epilepsy revealed upregulation of NKCC1 mRNA and downregulation of KCC2 transporters (Palma et al., 2006). Likewise, in a study by Huberfeld et al. on biopsies from human epileptic tissue, perturbed chloride homeostasis and GABAergic signaling were demonstrated, and bumetanide at doses that selectively block NKCC1, suppressed interictal activity (Huberfeld et al., 2007).

To design appropriate treatment strategies for these types of epilepsies, KCC2 and NKCC1 transporters should be considered as the targets (Payne et al., 2003). Brain-derived neurotrophic factor (BDNF) is one of the players in the regulation of $\mathrm{KCC} 2$ expression. Aguado et al. indicated that BDNF acting via tyrosine kinase B receptor (TrkB) can increase $\mathrm{KCC} 2$ expression during early development in embryos (Aguado et al., 2003). On the other hand, in a study by Rivera et al., KCC2 expression in mature central neurons was downregulated by BDNF (Rivera et al., 2004), indicating two modes of BDNF-mediated regulation of KCC2 expression.

Recently, Shulga et al. reported that BDNF increases $\mathrm{KCC} 2$ mRNA levels in axotomized central neurons. It suggested that injured neurons reverse their response to this neurotrophin by switching the BDNF-induced downregulation of KCC2 to upregulation (Shulga et al., 2008).

In our recent paper, we showed that both single high dose and multiple microinjections of BDNF can increase $\mathrm{KCC} 2$ expression. In addition, $\mathrm{NKCC} 1$ expression was downregulated by single high does injection of BDNF (Eftekhari et al., 2014). This study was aimed to evaluate the effect of BDNF treatment on epileptiform discharges in pilocarpine model of temporal lobe epilepsy.

\section{Methods \& Materials}

\subsection{Animals}

Adult male Wistar rats weighing 250-270 g were divided into sham, pilocarpine, single injection BDNF, and multiple injections BDNF groups. All experiments were housed in the controlled environment (7 AM-7 PM, light:dark cycle; $22 \pm 1^{\circ} \mathrm{C}$ ) with free access to food and water.

All experiments were performed in accordance with the Declaration of Helsinki, and the experiments were approved by the Ethics Committee of Iran University of Medical Sciences.

\subsection{Pilocarpine-induced Temporal Lobe Epilepsy}

Pilocarpine hydrochloride (Sigma, $350 \mathrm{mg} / \mathrm{kg}$ ), a muscarinic cholinergic agonist, was administered intraperitoneally (IP) to induce status epilepticus (SE). Animals were pretreated with the cholinergic antagonist scopolamine methyl nitrate (Sigma, $1 \mathrm{mg} / \mathrm{kg}$, IP) 30 minutes before the pilocarpine injection to reduce peripheral cholinergic effects (Ferhat et al., 2003). The animals' behavior was observed for several hours thereafter, and scored according to Racine's classification (Racine, 1972). Only rats that displayed SE (stages 3-5) for 3-4 hours were selected, and this period of robust seizures was terminated by a single injection of diazepam (7 mg/kg, IP). Animals were hand fed after SE until they could eat and drink on their own.

\subsection{Stereotaxic surgery}

Five days before the pilocarpine injection, animals were placed in a stereotaxic frame, under ketamine $(87 \mathrm{mg} / \mathrm{kg}$ 
IP) and xylazine (13 mg/kg IP) anesthesia. A guide cannula in the dorsal hippocampus (coordinates: $1.5 \mathrm{~mm}$ lateral and $1.7 \mathrm{~mm}$ posterior to bregma, $3.0 \mathrm{~mm}$ deep from dura) was inserted stereotaxically according to Pellegrino Atlas (Pellegrino LJ 1979). For ECoG recording, 2 silver electrodes on the parietal cortex and reference electrode were implanted on the nasal bulb. The animals in the multiple BDNF injection group received BDNF (Sigma, concentration: $1 \mu \mathrm{g}$ ) $\mu \mathrm{L}$, volume of injection:2.5 $\mu \mathrm{L}$ ) through the cannula on days $10,11,12$, and 13 after induction of status epilepticus.

The animals in the single BDNF injection group received BDNF with a single high-dose of $10 \mu \mathrm{L}$ BDNF with the same concentration on the $13^{\text {th }}$ day after induction of SE. Age-matched epileptic rats received the same amount of BDNF solvent, phosphate buffered saline in the sham group. All of the injections were bilateral. Since there was no difference between sham and pilocarpine groups, their data were pooled together.

\subsection{Checking electrocorticoencephalogram}

Animals were anesthetized with chlorhydrate $(400 \mathrm{mg} /$ $\mathrm{kg}$; Sigma-Aldrich) and silver electrodes were connected to the amplifier (EXT-02 F; NPI, Germany). Electrocorticoencephalogram (ECoG) was recorded for 1 hour twice weekly for 12 weeks after pilocarpine injection (Karimzadeh et al., 2012). Amplitude, frequency, and duration of epileptic activities were evaluated by AxoScope software.

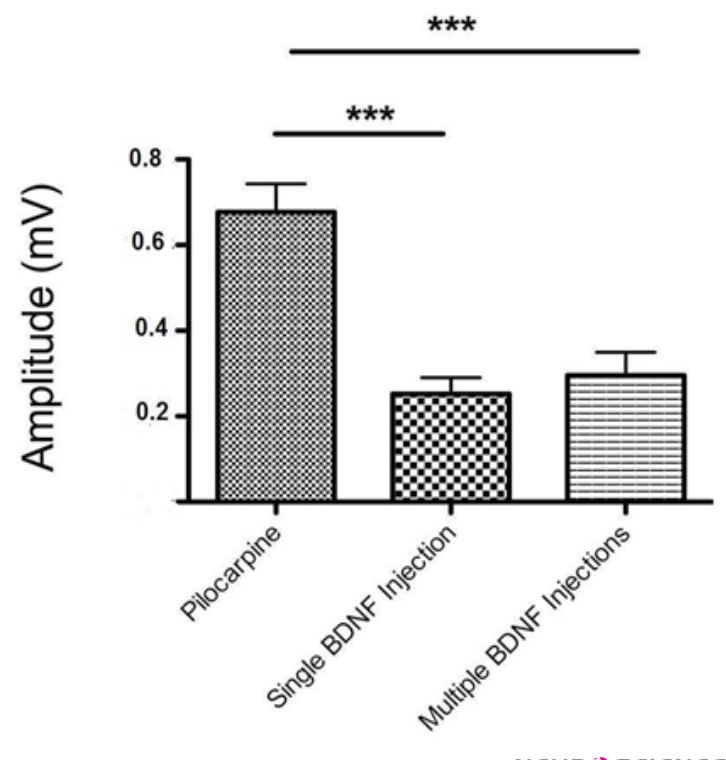

NEUR SCIENCE

Figure 1. The effect of BDNF treatment on the amplitude of epileptiform burst discharges induced by pilocarpine. The amplitude of spikes in both groups treated with BDNF was significantly lower than pilocarpine group (***indicates $\mathrm{P}<0.001$ ).

\section{Results}

The amplitude, frequency and duration of epileptiform burst discharges were compared among different groups. Both single and multiple microinjections of BDNF decreased amplitude and frequency of discharges compared to pilocarpine group $(\mathrm{P}<0.001$, Figures $1-2)$, but this changes were not permanent (Figure 3). In addition, there was no significant difference in duration of epileptiform burst discharges between BDNF-treated groups and pilocarpine group (Figure 4 ). The mean \pm SEM of all data were mentioned in the table 1.

\section{Discussion}

Various lines of evidence indicate that BDNF, a member of the neurotrophin family of growth factors (Leibrock et al., 1989), is associated with increased neuronal activity (Tanaka, Saito, \& Matsuki, 1997), and has been considered as a pro-epileptogenic factor (Rivera et al., 2004). Furthermore, according to several reports, the development of epilepsy can be attenuated or postponed by intrahippocampal delivery of BDNF (Koyama \& Ikegaya, 2005; Paradiso et al., 2009). To address these contrasting effects of BDNF, it is essential to clarify TLE pathogenesis.

While no definitive evidence exists to support a single mechanism for temporal lobe epileptogenesis, a large body of evidence suggests that depolarizing GABAergic sig-

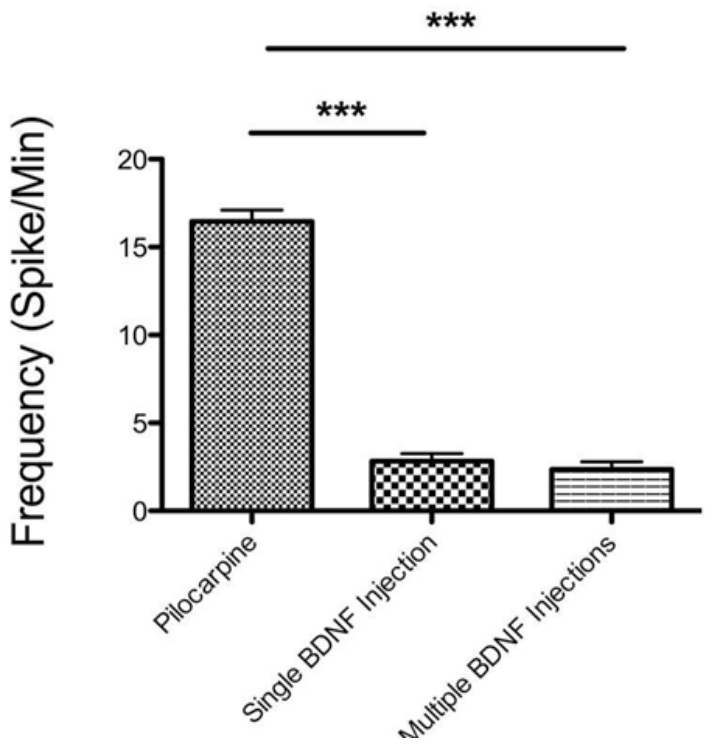

NEUR SCIENCE

Figure 2. The effect of BDNF treatment on the frequency of epileptiform burst discharges induced by pilocarpine. The frequency of spikes in both groups treated with BDNF was significantly lower than pilocarpine group (***indicates $\mathrm{P}<0.001)$. 

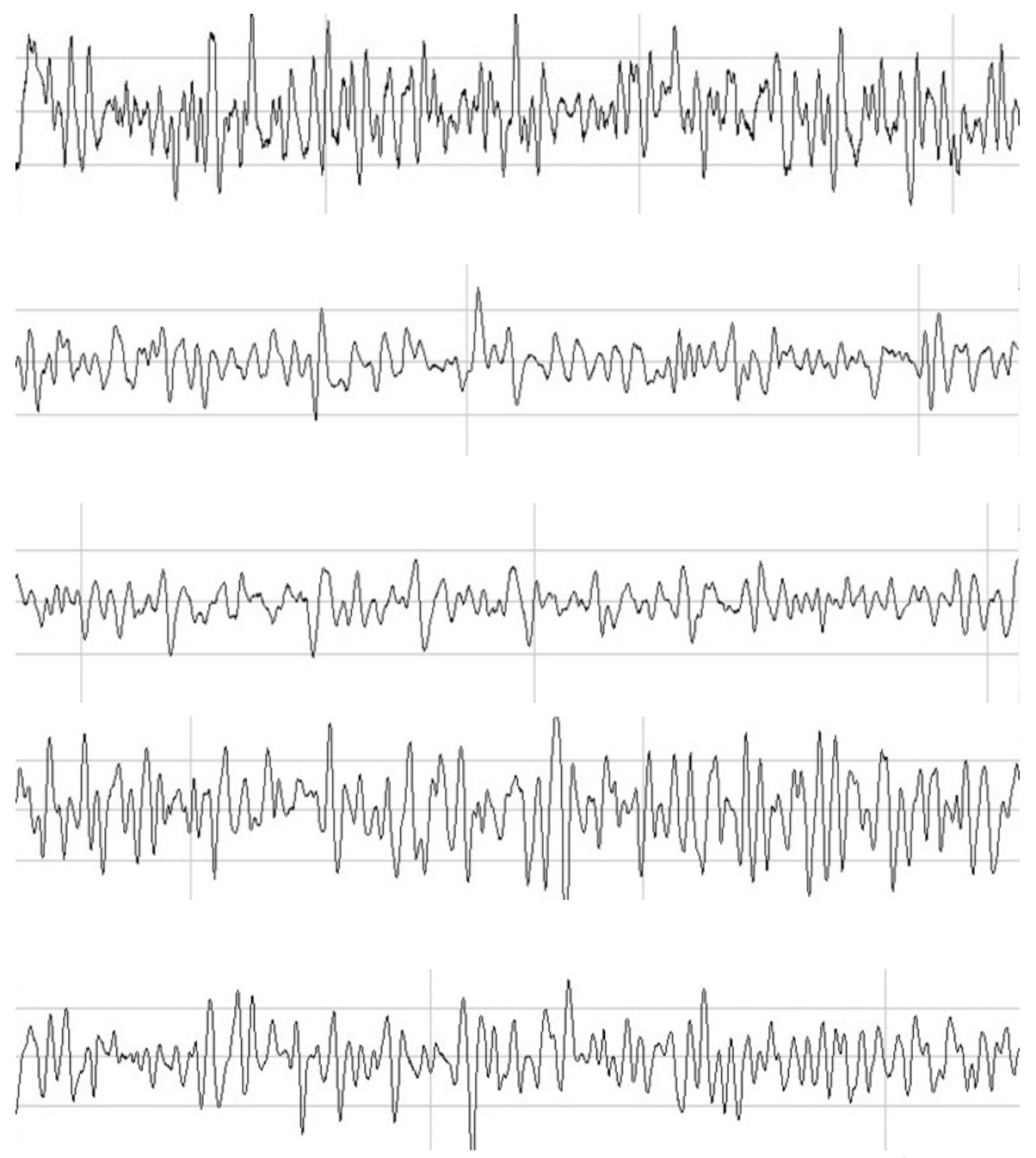

NEUR:SCIENCE

Figure 3. Recording of ECoG in all experiments. A: Pilocarpine group (2 weeks post-SE), B: Treated group with single high dose injection of BDNF (2 weeks post-SE), C: Treated group with multiple injections of BDNF (2 weeks post-SE), D: Treated group with single high dose injection of BDNF (12 weeks post-SE), E: Treated group with multiple injections of BDNF (12 weeks post-SE).

naling accompanied by altered expression of the chloride co-transporters KCC2 and NKCC1 contributes to epileptiform activity in animal models of epilepsies as well as human epileptic tissues (Cohen, Navarro, Clemenceau, Baulac, \& Miles, 2002; Dzhala et al., 2005; Palma et al., 2006; Huberfeld et al., 2007; Muñoz, Méndez, DeFelipe, \& Alvarez-Leefmans, 2007; Pathak et al., 2007). In our previous study, we showed that there is a notable imbalance between expression of 2 chloride transporters in an animal model of TLE (Eftekhari et al., 2014). This alteration in the expression of chloride transporters together with a depolarizing, immature-like response of GABA is in line with other pathophysiological conditions such as neuronal trauma, axotomy, and spinal cord injury (Nabekura et al.,

Table 1. The mean $\pm S E M$ of amplitude, frequency, and duration of spikes. There was significant decrease in the amplitude and frequency of spikes in BDNF-treated animals compared to pilocarpine groups ( ${ }^{* * *}$ indicates $\mathrm{P}<0.001$ ).

\begin{tabular}{|c|c|c|c|}
\hline Groups EEG & Pilocarpine & Single BDNF injection & Multiple BDNF injection \\
\hline Amplitude (mV) & $0.69 \pm 0.06$ & $0.24 \pm .02^{* * *}$ & $0.28 \pm 0.04^{* * *}$ \\
\hline Frequency (Spike/Min) & $16.45 \pm 0.06$ & $2.81 \pm 0.44 * * *$ & $2.36 \pm 0.43^{* * *}$ \\
\hline Duration (ms) & $5.35 \pm 1.17$ & $4.75 \pm 0.32$ & $4.33 \pm 0.57$ \\
\hline
\end{tabular}

NEUR:SCIENCE 


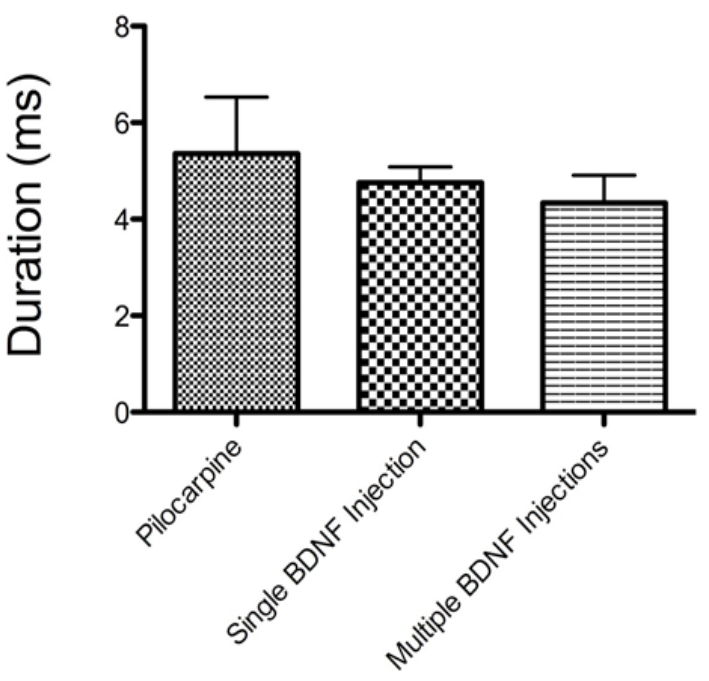

NEUR:SCIENCE

Figure 4. The effect of BDNF treatment on the duration of epileptiform burst discharges induced by pilocarpine. The duration of spikes in both groups treated with BDNF was not significantly different from pilocarpine group.

2002; Payne, Rivera, Voipio, \& Kaila, 2003; Shulga et al., 2008; Boulenguez et al., 2010). The depolarizing GABA-A mediated response during development is due to chloride uptake mediated by $\mathrm{NKCC} 1$, and the hyperpolarizing response in mature neurons is detectable when $\mathrm{KCC} 2$ expression increases causing a $\mathrm{Cl}^{-}$reduction (Rivera et al., 1999). Accumulating evidence indicate that upregulation of $\mathrm{KCC} 2$ is induced by BDNF leading to the functional maturation of GABA-A mediated responses (Aguado et al., 2003; Carmona et al., 2006; Ludwig et al., 2011). Ludwig et al. demonstrated that BDNF activates expression of the immediate early growth response 4 gene by induction of the TrkB/ mitogen activated protein kinase pathway, which stimulates KCC2b promoter activity (Ludwig et al., 2011). Furthermore, $\mathrm{KCC} 2$ expression is lower in the hippocampus of mice deficient in TrkB (Carmona et al., 2006) .On the other hand, in adult normal cortical neurons, BDNF downregulates $\mathrm{KCC} 2$ expression (Rivera et al., 2004), indicating two modes of BDNF-mediated regulation of $\mathrm{KCC} 2$ expression; Upregulation during development and downregulation in mature central neurons (Shulga et al., 2008).

Interestingly, Shulga et al. investigated the role of BDNF on $\mathrm{KCC} 2$ expression of axotomized corticospinal neurons, and demonstrated that injury activates a developmental-like mode of BDNF-mediated effects inducing a switch in the effect of BDNF on $\mathrm{KCC} 2$ expression from downregulatory to upregulatory (Shulga et al., 2008). Furthermore, Boulenguez et al. showed that while $\mathrm{KCC} 2$ is downregulated after spinal cord injury in rats, intrathecal injection of BDNF
(0.01 mg) can upregulate $\mathrm{KCC} 2$ expression 15 days after the injury (Boulenguez et al., 2010). In our recent study, we showed that application of either a single or multiple doses of BDNF, upregulated $\mathrm{KCC} 2$ expression (Eftekhari et al., 2014). In the current study, our data indicated that treatment with BDNF temporarily reduced the amplitude and frequency of epileptiform burst discharges. This effect might stabilize for a longer period of time if rats were infused continuously with BDNF using osmotic minipumps.

While recent studies point to NKCC1 inhibition as a promising strategy for TLE treatment (Huberfeld et al., 2007; Brandt, Nozadze, Heuchert, Rattka, \& Löscher, 2010; Eftekhari et al., 2013), an increase in KCC2 expression can be considered an alternative treatment to bumetanide application. If we want to focus on $\mathrm{KCC} 2$ activation as a TLE treatment strategy, it is essential to find out for how long after induction of TLE, BDNF can manage to upregulate $\mathrm{KCC} 2$, keeping its immature-state function. On the other hand, as applying exogenous BDNF is not practical and amenable for human patients, future experiments are needed to modify $\mathrm{KCC} 2$ expression via endogenous BDNF. Although, Shulga et al. mentioned in their study that the endogenous levels of BDNF were not enough to upregulate KCC2 (Shulga et al., 2008), more recent studies point to the upregulation of endogenous BDNF by application of some hormones like thyroxin (Shulga et al., 2009) and statins like simvastatin (Han et al., 2011) in injured neurons. Future studies are warranted to assess any possible therapeutic effects of such agents on temporal lobe epilepsies via inducing endogenous BDNF.

\section{References}

Aguado, F., Carmona, M. A., Pozas, E., Aguiló, A., Martínez-Guijarro, F. J., Alcantara, S., et al. (2003). BDNF regulates spontaneous correlated activity at early developmental stages by increasing synaptogenesis and expression of the $\mathrm{K}+/ \mathrm{Cl}$-co-transporter KCC2. Development, 130(7), 1267-1280.

Ben-Ari, Y. (2002). Excitatory actions of GABA during development: the nature of the nurture. Nature Reviews Neuroscience, 3(9), 728-739.

Ben-Ari, Y., Gaiarsa, J. L., Tyzio, R., \& Khazipov, R. (2007). GABA: a pioneer transmitter that excites immature neurons and generates primitive oscillations. Physiological Reviews, 87(4), 1215-1284.

Boulenguez, P., Liabeuf, S., Bos, R., Bras, H., Jean-Xavier, C., Brocard, C., et al. (2010). Down-regulation of the potassium-chloride cotransporter KCC2 contributes to spasticity after spinal cord injury. Nature Medicine, 16(3), 302-307.

Brandt, C., Nozadze, M., Heuchert, N., Rattka, M., \& Löscher, W. (2010). Disease-modifying effects of phenobarbital and the 
$\mathrm{NKCC1}$ inhibitor bumetanide in the pilocarpine model of temporal lobe epilepsy. Journal of Neuroscience, 30(25), 8602-8612.

Carmona, M. A., Pozas, E., Martínez, A., Espinosa-Parrilla, J. F., Soriano, E., \& Aguado, F. (2006). Age-dependent spontaneous hyperexcitability and impairment of GABAergic function in the hippocampus of mice lacking trkB. Cerebral Cortex, 16(1), 47-63.

Cohen, I., Navarro, V., Clemenceau, S., Baulac, M., \& Miles, R. (2002). On the origin of interictal activity in human temporal lobe epilepsy in vitro. Science, 298(5597), 1418-1421.

Cohen, I., Navarro, V., Duigou, C., \& Miles, R. (2003). Mesial temporal lobe epilepsy: A pathological replay of developmental mechanisms? Biology of the Cell, 95(6), 329-333.

Dzhala, V. I., Talos, D. M., Sdrulla, D. A., Brumback, A. C., Mathews, G. C., Benke, T. A., et al. (2005). NKCC1 transporter facilitates seizures in the developing brain. Nature Medicine, 11(11), 1205-1213.

Eftekhari, S., Mehvari Habibabadi, J., Najafi Ziarani, M., Hashem Fesharaki, S. S., Gharakhani, M., Mostafavi, H., et al. (2013). Bumetanide reduces seizure frequency in patients with temporal lobe epilepsy. Epilepsia, 54(1), e9-e12.

Eftekhari, S., Mehrabi, S., Soleimani, M., Hassanzadeh, G., Rahmanzadeh, R., Hadjighassem, M. R., et al. (2014). BDNF modifies hippocampal KCC2 and NKCC1 expression in a temporal lobe epilepsy model. Acta Neurobiologiae Experimentalis, 74(3), 276-287.

Engel, J. (2001). Mesial temporal lobe epilepsy: what have we learned? Neuroscientist, 7(4), 340-352.

Ferhat, L., Esclapez, M., Represa, A., Fattoum, A., Shirao, T., \& BenAri, Y. (2003). Increased levels of acidic calponin during dendritic spine plasticity after pilocarpine-induced seizures. Hippocampus, 13(7), 845-858.

Han, X., Yang, N., Xu, Y., Zhu, J., Chen, Z., Liu, Z., et al. (2011). Simvastatin treatment improves functional recovery after experimental spinal cord injury by upregulating the expression of BDNF and GDNF. Neuroscience Letters, 487(3), 255-259.

Huberfeld, G., Wittner, L., Clemenceau, S., Baulac, M., Kaila, K., Miles, R., et al. (2007). Perturbed chloride homeostasis and GABAergic signaling in human temporal lobe epilepsy. Journal of Neuroscience, 27(37), 9866-9873.

Karimzadeh, F., Hosseini, M., Mangeng, D., Alavi, H., Hassanzadeh, G. R., Bayat, M., et al. (2012). Anticonvulsant and neuroprotective effects of Pimpinella anisum in rat brain. BMC Complementary and Alternative Medicine, 12(1), 76.

Koyama, R., \& Ikegaya, Y. (2005). To BDNF or not to BDNF: that is the epileptic hippocampus. Neuroscientist, 11(4), 282-287.

Leibrock, J., Lottspeich, F., Hohn, A., Hofer, M., Hengerer, B., Masiakowski, P., et al. (1989). Molecular cloning and expression of brain-derived neurotrophic factor. Nature, 341, 149-152.

Ludwig, A., Uvarov, P., Soni, S., Thomas-Crusells, J., Airaksinen, M. S., \& Rivera, C. (2011). Early growth response 4 mediates BDNF induction of potassium chloride cotransporter 2 transcription. Journal of Neuroscience, 31(2), 644-649.

Muñoz, A., Méndez, P., DeFelipe, J., \& Alvarez-Leefmans, F. J. (2007). Cation-chloride cotransporters and GABA-ergic innervation in the human epileptic hippocampus. Epilepsia, 48(4), 663673.
Nabekura, J., Ueno, T., Okabe, A., Furuta, A., Iwaki, T., ShimizuOkabe, C., et al. (2002). Reduction of KCC2 Expression and GABAAReceptor-Mediated Excitation after In Vivo Axonal Injury. Journal of Neuroscience, 22(11), 4412-4417.

Okabe, A., Ohno, K., Toyoda, H., Yokokura, M., Sato, K., \& Fukuda, A. (2002). Amygdala kindling induces upregulation of mRNA for $\mathrm{NKCC1}$, a Na+, $\mathrm{K}+-2 \mathrm{Cl}$ - cotransporter, in the rat piriform cortex. Neuroscience Research, 44(2), 225-229.

Palma, E., Amici, M., Sobrero, F., Spinelli, G., Di Angelantonio, S. Ragozzino, D., et al. (2006). Anomalous levels of $\mathrm{Cl}$ - transporters in the hippocampal subiculum from temporal lobe epilepsy patients make GABA excitatory. Proceedings of the National Academy of Sciences, 103(22), 8465-8468.

Paradiso, B., Marconi, P., Zucchini, S., Berto, E., Binaschi, A., Bozac, A., et al. (2009). Localized delivery of fibroblast growth factor-2 and brain-derived neurotrophic factor reduces spontaneous seizures in an epilepsy model. Proceedings of the National Academy of Sciences, 106(17), 7191-7196.

Pathak, H. R., Weissinger, F., Terunuma, M., Carlson, G. C., Hsu, F. C., Moss, S. J., et al. (2007). Disrupted dentate granule cell chloride regulation enhances synaptic excitability during development of temporal lobe epilepsy. Journal of Neuroscience, 27(51), 14012 14022 .

Payne, J. A., Rivera, C., Voipio, J., \& Kaila, K. (2003). Cation-chloride co-transporters in neuronal communication, development and trauma. Trends in Neurosciences, 26(4), 199-206.

Rivera, C., Voipio, J., Payne, J. A., Ruusuvuori, E., Lahtinen, H., Lamsa, K., et al. (1999). The K+/Cl- co-transporter KCC2 renders GABA hyperpolarizing during neuronal maturation. Nature, 397(6716), 251-255.

Rivera, C., Voipio, J., Thomas-Crusells, J., Li, H., Emri, Z., Sipilä, S., et al. (2004). Mechanism of activity-dependent downregulation of the neuron-specific $\mathrm{K}-\mathrm{Cl}$ cotransporter KCC2. Journal of Neuroscience, 24(19), 4683-4691.

Shulga, A., Blaesse, A., Kysenius, K., Huttunen, H. J., Tanhuanpää, K., Saarma, M., et al. (2009). Thyroxin regulates BDNF expression to promote survival of injured neurons. Molecular and Cellular Neuroscience, 42(4), 408-418.

Shulga, A., Thomas-Crusells, J., Sigl, T., Blaesse, A., Mestres, P., Meyer, M., et al. (2008). Posttraumatic GABA(A)-mediated $[\mathrm{Ca} 2+] \mathrm{i}$ increase is essential for the induction of brain-derived neurotrophic factor-dependent survival of mature central neurons. Journal of Neuroscience, 28(27), 6996-7005.

Tanaka, T., Saito, H., \& Matsuki, N. (1997). Inhibition of GABAA synaptic responses by brain-derived neurotrophic factor (BDNF) in rat hippocampus. Journal of Neuroscience, 17(9), 2959-2966.

Woo, N. S., Lu, J., England, R., McClellan, R., Dufour, S., Mount, D. B., et al. (2002). Hyperexcitability and epilepsy associated with disruption of the mouse neuronal-specific $\mathrm{K}-\mathrm{Cl}$ cotransporter gene. Hippocampus, 12(2), 258-268 